\title{
Tube Thoracostomy
}

National Cancer Institute

\section{Source}

National Cancer Institute. Tube Thoracostomy. NCI Thesaurus. Code C148463.

A surgical procedure in which a chest tube is inserted between the ribs to drain fluid or air from the pleural cavity. 